\title{
Uniform Continuity Properties of Preference Relations
}

\author{
Douglas S. Bridges
}

\begin{abstract}
The anti-Specker property, a constructive version of sequential compactness, is used to prove constructively that a pointwise continuous, order-dense preference relation on a compact metric space is uniformly sequentially continuous. It is then shown that Ishihara's principle BD- $\mathbb{N}$ implies that a uniformly sequentially continuous, order-dense preference relation on a separable metric space is uniformly continuous. Converses of these two theorems are also proved.
\end{abstract}

\section{Introduction}

This paper continues the constructive reverse-mathematical investigation of various continuity properties of preference relations on a metric space, begun in [2]. In that paper we discussed sequential and pointwise continuity; in this one we deal with two classically equivalent, but (as we shall see) constructively distinct, notions of uniform continuity for preferences. As in our earlier paper, we work here within the framework of Bishop-style constructive mathematics (BISH); in other words, we use only intuitionistic logic and an appropriate set theory such as that in [1], [15], or [20]. Moreover, we work in an informal setting, not relative to some precise formal system; this is in keeping with our earlier papers and with normal practice in Bishopstyle constructive mathematics.

We refer the reader to [4], [5], [12], and [22] for background material on constructive mathematics, and to [6], [7], and [8] for more information about preference, utility, and demand in a constructive setting.

Let $X$ be a set that is inhabited-that is, in which we can construct an element. A binary relation $\succ$ on $X$ is called a preference relation if it satisfies the two conditions:

$$
\begin{array}{ll}
\mathbf{P}_{1} & \forall x, y \in X(x \succ y \Rightarrow \neg(y \succ x)) ; \\
\mathbf{P}_{2} & \forall x, y \in X\left(x \succ y \Rightarrow \forall_{z \in X}(x \succ z \vee z \succ y)\right) .
\end{array}
$$


We then have the corresponding preference-indifference relation $\succcurlyeq$ defined by

$$
\forall_{x, y \in X}\left(x \succcurlyeq y \Leftrightarrow \forall_{z \in X}(y \succ z \Rightarrow x \succ z)\right) .
$$

Note that

$$
x \succcurlyeq y \Leftrightarrow \neg(y \succ x),
$$

and that if $x \succ y \succcurlyeq z$ or $x \succcurlyeq y \succ z$, then, by $\mathbf{P}_{2}$, we have $x \succ z$. We say that $\succ$ is order-dense (in $X$ ) if

$$
\forall_{x, y \in X}\left(x \succ y \Rightarrow \exists_{z \in X}(x \succ z \succ y)\right) .
$$

We are particularly interested in the case where $(X, \rho)$ is a metric space, in which case we have a standard inequality relation $\neq$ defined by

$$
\forall_{x, y \in X}(x \neq y \Leftrightarrow \rho(x, y)>0) .
$$

We say that a preference relation $\succ$ on $X$ is

$\triangleright$ sequentially continuous if for each $x \in X$, each sequence $\left(x_{n}\right)_{n \geqslant 1}$ of points of $X$ converging to $x$, and each $a \in X$,

$$
x>a \Rightarrow \exists_{N} \forall_{n \geqslant N}\left(x_{n} \succ a\right)
$$

and

$$
a \succ x \Rightarrow \exists_{N} \forall_{n \geqslant N}\left(a \succ x_{n}\right) ;
$$

$\triangleright$ pointwise continuous if for each $x \in X$, the sets

$$
\begin{aligned}
& (\leftarrow, x)=\{y \in X: x \succ y\}, \\
& (x, \rightarrow)=\{y \in X: y \succ x\}
\end{aligned}
$$

are open in $X$.

These properties were studied in [2]. In the present paper we look at two uniform continuity properties of a preference relation $\succ$ on a metric space $X$. We say that $\succ$ is

$\triangleright$ uniformly sequentially continuous on $X$ if for all $a, b \in X$ with $a \succ b$, and all sequences $\left(x_{n}\right)_{n \geqslant 1},\left(x_{n}^{\prime}\right)_{n \geqslant 1}$ in $X$ such that $\rho\left(x_{n}, x_{n}^{\prime}\right) \rightarrow 0$ as $n \rightarrow \infty$, there exists $N$ such that for all $n \geqslant N$, either $a \succ x_{n}$ or $x_{n}^{\prime} \succ b$;

$\triangleright$ uniformly continuous on $X$ if for all $a, b \in X$ with $a \succ b$ there exists $\delta>0$ such that for all $x, x^{\prime} \in X$ with $\rho\left(x, x^{\prime}\right)<\delta$, either $a \succ x$ or $x^{\prime} \succ b$.

Clearly, uniform continuity implies uniform sequential continuity. The converse holds classically: for if $\succ$ is not uniformly continuous on $X$, then (classically) there exist sequences $\left(x_{n}\right)_{n \geqslant 1},\left(x_{n}^{\prime}\right)_{n \geqslant 1}$ in $X$ such that $\rho\left(x_{n}, x_{n}^{\prime}\right) \rightarrow 0$ as $n \rightarrow \infty$, but $x_{n} \succcurlyeq a$ and $b \succcurlyeq x_{n}^{\prime}$ for each $n$; whence $\succ$ is not uniformly sequentially continuous on $X$. As we shall see, uniform sequential continuity is constructively weaker than uniform continuity. This is in keeping with the parallel situation for functions between metric spaces [13].

Suppose that the preference relation $\succ$ on the metric space $X$ is represented by a utility function: that is, a mapping $u: X \rightarrow \mathbb{R}$ such that

$$
\forall_{x, y \in X}(x \succ y \Leftrightarrow u(x)>u(y)) .
$$

If $u$ satisfies the following property of uniform sequential continuity (for a function) on $X$, introduced in [13], then the preference relation $\succ$ is uniformly sequentially continuous on $X$ : 


$$
\begin{aligned}
& \text { If }\left(x_{n}\right)_{n \geqslant 1},\left(x_{n}^{\prime}\right)_{n \geqslant 1} \text { are sequences in } X \text { such that } \rho\left(x_{n}, x_{n}^{\prime}\right) \rightarrow 0 \text { as } \\
& n \rightarrow \infty \text {, then }\left|u\left(x_{n}\right)-u\left(x_{n}^{\prime}\right)\right| \rightarrow 0 \text { as } n \rightarrow \infty .
\end{aligned}
$$

Suppose that $u$ is uniformly sequentially continuous in this sense. Consider sequences $\left(x_{n}\right)_{n \geqslant 1},\left(x_{n}^{\prime}\right)_{n \geqslant 1}$ in $X$ such that $\rho\left(x_{n}, x_{n}^{\prime}\right) \rightarrow 0$ as $n \rightarrow \infty$, and points $a, b$ of $X$ such that $a \succ b$ and therefore $u(a)>u(b)$. Setting $\varepsilon=\frac{1}{2}(u(a)-u(b))$, compute $N$ such that $\left|u\left(x_{n}\right)-u\left(x_{n}^{\prime}\right)\right|<\varepsilon$ for all $n \geqslant N$. For such $n$, either $u\left(x_{n}\right)<u(a)$ or $u\left(x_{n}\right)>u(a)-\varepsilon$. In the first case, $a \succ x_{n}$; in the second,

$$
u\left(x_{n}^{\prime}\right) \geqslant u\left(x_{n}\right)-\left|u\left(x_{n}\right)-u\left(x_{n}^{\prime}\right)\right|>u(a)-2 \varepsilon=u(b),
$$

so $x_{n}^{\prime} \succ b$. This completes the proof that $\succ$ is uniformly sequentially continuous on $X$. It is even easier to prove that if $u$ is uniformly continuous on $X$, then $\succ$ also is uniformly continuous on $X$.

Note that a utility function $u$ representing a uniformly sequentially continuous preference relation $\succ$ on a metric space need not be uniformly sequentially continuous: consider the space

the preference relation

$$
X=\{0\} \cup\left\{\frac{1}{n}: n \in \mathbb{N}^{+}\right\}
$$

$$
\succ=\left\{\left(\frac{1}{n}, 0\right): n \in \mathbb{N}^{+}\right\} \cup\left\{\left(\frac{1}{m}, \frac{1}{n}\right): m, n \in \mathbb{N}^{+} ; m<n\right\}
$$

on $X$, and the representing utility function $u: X \rightarrow \mathbb{R}$ defined by

$$
u(x)= \begin{cases}x & \text { if } x>0 \\ -1 & \text { if } x=0\end{cases}
$$

Incidentally, the intermediate value theorem ([10], Chapter 3, Section 2) shows that if $X$ is a convex subset of a normed space, and $\succ$ is represented by a sequentially continuous utility function on $X$, then $\succ$ is order-dense.

Recall that in BISH a metric space is

$\triangleright$ compact if it is complete and totally bounded, and

$\triangleright$ locally compact if every bounded subset is contained in a compact set.

If $X$ is a locally compact metric space and the preference relation $\succ$ is uniformly continuous on each compact subspace of $X$, then $\succ$ is pointwise continuous. For in that case, if $a \succ b$, we can construct a compact set $K \subset X$ such that $x \in K$ if either $\rho(a, x) \leqslant 1$ or $\rho(b, x) \leqslant 1$. Choosing $\delta<1$ as in the definition of "uniformly continuous" above, we see that if $\rho(a, x)<\delta$, then either $a \succ a$ or, as must be the case, $x \succ b$. It follows that $(b, \rightarrow)$ is open. Similarly, $(\leftarrow, a)$ is open.

We are interested in constructive answers to these two questions:

Under what conditions does the pointwise continuity of $\succ$ imply its uniform sequential continuity?

Under what conditions does the uniform sequential continuity of $\succ$ imply its uniform continuity?

The reader may be wondering by now why we don't work with utility-function representations of a preference relation and apply already-known corresponding results about continuous functions, such as those in [16] and [13]. The reason is simple: although there are constructive utility-function representations of certain preferences, 
there is a recursive counterexample to Debreu's classical theorem [14] on the existence of utility functions; see [6], [8], and [11] for more on these matters. We hope that a close study of the connections between various continuity properties of a preference relation may actually lead to a better understanding of the constructive representability of such functions by utility functions.

\section{Pointwise and Uniform Sequential Continuity}

To answer the first of our questions, we use a property classically equivalent to sequential compactness. First we recall that a sequence $\left(z_{n}\right)_{n \geqslant 1}$ in a metric space $Z$ is eventually bounded away from the subset $X$ of $Z$ if there exist $N$ and $\delta>0$ such that $\rho\left(x, z_{n}\right)>\delta$ for all $x \in X$ and all $n \geqslant N$. If $X=\{x\}$ is a singleton, we say that the sequence is eventually bounded away from the point $x$. This brings us to the anti-Specker property for $X$ :

AS If $X$ is embedded isometrically as a subspace of a metric space $Z$, then every sequence in $Z$ that is eventually bounded away from each point of $X$ is eventually bounded away from $X$.

It is not hard to show that this property is independent of the space $Z$ in which $X$ is isometrically embedded, and that $\mathbf{A S}$ is classically equivalent to the BolzanoWeierstraß property for $X$. In the case $Z=\mathbb{R}$ and $X=[0,1]$,

(i) AS is the antithesis of Specker's recursive counterexample [21] to the monotone convergence theorem for sequences;

(ii) as is shown in [3], $\mathbf{A S}$ is equivalent, relative to $\mathbf{B I S H}$, to a version of Brouwer's fan theorem.

For more on the use of AS in constructive reverse mathematics see also [9].

Recalling that the metric complement of a subset $X$ of a metric space $Z$ is the set

$$
Z-X=\left\{z \in Z: \exists_{r>0} \forall_{x \in X}(\rho(z, x) \geqslant r)\right\},
$$

we can now state our first main theorem.

Theorem 2.1 Let $\succ$ be a pointwise continuous, order-dense preference relation on a metric space $X$ that has the anti-Specker property. Then $\succ$ is uniformly sequentially continuous on $X$.

Proof Embed $X$ in a metric space $Z$ such that there exists $\zeta \in Z-X$, and compute $r>0$ such that $\rho(x, \zeta) \geqslant r$ for all $x \in X$. Given $a, b \in X$ with $a \succ b$, pick $a_{0}, b_{0} \in X$ such that $a \succ a_{0} \succ b_{0} \succ b$. Let $\left(x_{n}\right)_{n \geqslant 1},\left(x_{n}^{\prime}\right)_{n \geqslant 1}$ be sequences in $X$ such that $\rho\left(x_{n}, x_{n}^{\prime}\right) \rightarrow 0$ as $n \rightarrow \infty$, and, noting $\mathbf{P}_{2}$, construct a binary sequence $\left(\lambda_{n}\right)_{n \geqslant 1}$ such that

$$
\begin{aligned}
& \lambda_{n}=0 \Rightarrow a \succ x_{n} \vee x_{n}^{\prime} \succ b, \\
& \lambda_{n}=1 \Rightarrow x_{n} \succ a_{0} \wedge b_{0} \succ x_{n}^{\prime} .
\end{aligned}
$$

If $\lambda_{n}=0$, set $s_{n}=\zeta$; if $\lambda_{n}=1$, set $s_{n}=x_{n}$. Given $x \in X$, we have either $a_{0} \succ x$ or $x \succ b_{0}$. In the first case, since $\succ$ is pointwise continuous at $a_{0}$, there exists $\delta>0$ such that if $x^{\prime} \in X$ and $\rho\left(x, x^{\prime}\right)<\delta$, then $a_{0} \succ x^{\prime}$. Compute $N$ such that $\rho\left(x_{n}, x_{n}^{\prime}\right)<\delta$ for all $n \geqslant N$. Suppose that, for some such $n$,

$$
\rho\left(x, s_{n}\right)<\min \left\{r, \frac{\delta}{2}\right\} .
$$


Then $s_{n} \neq \zeta$, so $\lambda_{n}=1, s_{n}=x_{n}$, and $x_{n} \succ a_{0}$. But $\rho\left(x, x_{n}\right)=\rho\left(x, s_{n}\right)<\delta / 2$, so $a_{0} \succ x_{n}-$ a contradiction, from which we conclude that, after all,

$$
\forall_{n} \geqslant N\left(\rho\left(x, s_{n}\right) \geqslant \min \left\{r, \frac{\delta}{2}\right\}\right) .
$$

In the case $x \succ b_{0}$, we compute $\delta>0$ such that if $x^{\prime} \in X$ and $\rho\left(x, x^{\prime}\right)<\delta$, then $y \succ b_{0}$. We also compute $N$ such that $\rho\left(x_{n}, x_{n}^{\prime}\right)<\delta / 2$ for all $n \geqslant N$. For such $n$, if (2) holds, then $s_{n} \neq \zeta$, so $\lambda_{n}=1, s_{n}=x_{n}$, and $b_{0} \succ x_{n}^{\prime}$. Also,

$$
\rho\left(x, x_{n}^{\prime}\right) \leqslant \rho\left(x, s_{n}\right)+\rho\left(x_{n}, x_{n}^{\prime}\right)<\delta,
$$

so (by our choice of $\delta$ ) $x_{n}^{\prime} \succ b_{0}$-another contradiction, from which we conclude that formula (3) holds in this case also. Thus the sequence $\left(s_{n}\right)_{n \geqslant 1}$ is eventually bounded away from each $x \in X$. Applying AS, compute a positive integer $v$ and $\gamma>0$ such that $\rho\left(x, s_{n}\right)>\gamma$ for all $x \in X$ and all $n \geqslant \nu$. It follows that $\lambda_{n} \neq 1$, and hence that either $a \succ x_{n}$ or $x_{n}^{\prime} \succ b$, for all such $n$.

The following definition will lead to a converse of Theorem 2.1 in the case $X=\mathbb{R}$. For each $t \in \mathbb{R}$ and each $\delta>0$ define the corresponding spike function to be the unique uniformly continuous function $s(t, \delta,):. \mathbb{R} \rightarrow \mathbb{R}$ with the following properties:

(a) $s(t, \delta, t)=1$,

(b) $s(t, \delta, x)=0$ whenever $|x-t|>\delta$, and

(c) $s(t, \delta, \cdot)$ is linear in each of the intervals $[t-\delta, t]$ and $[t, t+\delta]$.

Theorem 2.2 Suppose that every pointwise continuous, order-dense preference relation on $\mathbb{R}$ is uniformly sequentially continuous. Then every sequence in $\mathbb{R}$ that is eventually bounded away from each point of $[0,1]$ is eventually bounded away from the interval $[0,1]$.

Proof Let $\left(r_{n}\right)_{n \geqslant 1}$ be a sequence of real numbers eventually bounded away from each point of [0, 1]. In view of [9], Lemma 4, we may assume that $r_{m} \neq r_{n}$ whenever $m \neq n$. Replacing the sequence $\left(r_{n}\right)_{n \geqslant 1}$ by one of its tails, if necessary, we may further assume that there exists $\delta_{0} \in\left(0, \frac{1}{4}\right)$ such that $\left|r_{n}\right|>\delta_{0}$ and $\left|r_{n}-1\right|>\delta_{0}$ for all $n$. We may assume, finally, that $r_{1}=1 / 2$. Setting $n_{0}=1$, we can construct, as in the proof of [9], Theorem 5, a sequence $\left(\delta_{k}\right)_{k \geqslant 0}$ of positive numbers and a strictly increasing sequence $\left(n_{k}\right)_{k \geqslant 0}$ of positive integers such that the following hold for each $k \geqslant 1$ :

(i) $\delta_{k} \leqslant \min \left\{2^{-k-1}, \delta_{k-1}\right\}$;

(ii) $\left|r_{n}-r_{k}\right| \geqslant 2 \delta_{k}$ for all $n>n_{k}$.

It follows from [9], Lemma 3, that

$$
u(x)=\sum_{k=1}^{\infty} s\left(r_{k}, \delta_{k}, x\right)
$$

defines a pointwise continuous function on $[0,1]$ such that the supports of the terms of the series are disjoint. Now define a (clearly pointwise continuous) preference relation $\succ$ on $[0,1]$ by

$$
x>y \Leftrightarrow u(x)>u(y) .
$$

Note that $\succ$ is order-dense, by the intermediate value theorem applied to $u$ on $\left[r_{1}, r_{1}+\delta_{1}\right]$. Our choice of $\delta_{0}$ ensures that, for each $n \geqslant 1$, either $r_{n} \in\left(\delta_{0}, 1-\delta_{0}\right)$ 
or $\rho\left(r_{n},[0,1]\right)>\delta_{0}$. In the first case, setting $x_{n}=r_{n}$ and $x_{n}^{\prime}=r_{n}+\delta_{n}$, we see that both $x_{n}$ and $x_{n}^{\prime}$ are in $[0,1],\left|x_{n}-x_{n}^{\prime}\right| \leqslant 2^{-n-1}, u\left(x_{n}\right)=1$, and $u\left(x_{n}^{\prime}\right)=0$. In the case $\rho\left(r_{n},[0,1]\right)>\delta_{0}$, we set $x_{n}=x_{n}^{\prime}=1$. Altogether, we obtain sequences $\left(x_{n}\right)_{n \geqslant 1},\left(x_{n}^{\prime}\right)_{n \geqslant 1}$ in $[0,1]$ such that $\left|x_{n}-x_{n}^{\prime}\right| \rightarrow 0$ as $n \rightarrow \infty$. By hypothesis, the preference relation $\succ$ is uniformly sequentially continuous. Since $x_{1}=r_{1}$ and $x_{1}^{\prime}=r_{1}+\delta_{1}$ (both points being in $\left(\delta_{0}, 1-\delta_{0}\right)$ ), we have $x_{1} \succ x_{1}^{\prime}$; so there exists $N>1$ such that for each $n \geqslant N$, either $x_{1} \succ x_{n}$ or $x_{n}^{\prime} \succ x_{1}^{\prime}$; whence either $u\left(x_{1}\right)>u\left(x_{n}\right)$ or $u\left(x_{n}^{\prime}\right)>u\left(x_{1}^{\prime}\right)$. For such a value of $n$, if $r_{n} \in\left(\delta_{0}, 1-\delta_{0}\right)$, then $u\left(x_{n}\right)=1=u\left(x_{1}\right)$ and $u\left(x_{n}^{\prime}\right)=0=u\left(x_{1}^{\prime}\right)$, so $x_{n} \succcurlyeq x_{1}$ and $x_{1}^{\prime} \succcurlyeq x_{n}^{\prime}$, a contradiction. Hence $\rho\left(r_{n},[0,1]\right)>\delta_{0}$ for all $n \geqslant N$.

Thus we see from Theorems 2.1 and 2.2 that the anti-Specker property is equivalent to the passage from pointwise continuity to uniform sequential continuity for orderdense preference relations on metric spaces.

\section{Uniform Sequential, and Uniform, Continuity}

Before coming to our third theorem, we define a set $S$ of positive integers to be pseudobounded if, for each sequence $\left(s_{n}\right)_{n \geqslant 1}$ in $S, n^{-1} s_{n} \rightarrow 0$ as $n \rightarrow \infty$. The paper [2] explored the connection between the principle

BD- $\mathbb{N}$ Every inhabited, countable, pseudobounded set of positive integers is bounded,

introduced by Ishihara in [16], and the statement

Every sequentially continuous, order-dense preference relation on a complete, separable metric space is pointwise continuous.

Note that BD- $\mathbb{N}$ holds classically and in both the intuitionistic and recursive models of constructive mathematics (see [16]), is unprovable within a natural formal system for BISH (see [19]) and—not least in the work of Ishihara (for example, [16], [17], [18]) — has already established its significance for constructive reverse mathematics.

Theorem 3.1 Assume BD-N. Then every uniformly sequentially continuous, orderdense preference relation on a separable metric space is uniformly continuous.

Proof Let $X$ be a separable metric space, $\left(q_{n}\right)_{n \geqslant 1}$ a dense sequence in $X$, and $\succ$ a uniformly sequentially continuous, order-dense preference relation on $X$. Let $a, a_{0}, a_{1}, b, b_{0}, b_{1}$ be points of $X$ such that $a \succ a_{0} \succ a_{1} \succ b_{1} \succ b_{0} \succ b$. Construct a mapping $\varphi: \mathbb{N} \times \mathbb{N} \times \mathbb{N} \rightarrow\{0,1\}$ such that

$$
\begin{aligned}
& \triangleright \text { if } \varphi(i, j, k)=0, \text { then } \rho\left(q_{i}, q_{j}\right)<1 / k, q_{i} \succ a_{1} \text {, and } b_{1} \succ q_{j} ; \\
& \triangleright \text { if } \varphi(i, j, k)=1 \text {, then } \rho\left(q_{i}, q_{j}\right)>1 /(k+1) \text { or } a_{0} \succ q_{i} \text { or } q_{j} \succ b_{0} .
\end{aligned}
$$

Let

$$
S=\{0\} \cup\left\{k: \exists_{i, j}(\varphi(i, j, k)=0)\right\} .
$$

Then $S$ is an inhabited countable subset of $\mathbb{N}^{+}$. We prove that $S$ is pseudobounded. To that end, let $\left(s_{n}\right)_{n \geqslant 1}$ be any sequence in $S$, and let $\varepsilon>0$. Construct a binary sequence $\left(\lambda_{n}\right)_{n} \geqslant 1$ such that

$$
\begin{aligned}
& \lambda_{n}=0 \Rightarrow n^{-1} s_{n}<\varepsilon, \\
& \lambda_{n}=1 \Rightarrow n^{-1} s_{n}>\varepsilon / 2 .
\end{aligned}
$$


If $\lambda_{n}=0$, set $x_{n}=q_{1}=x_{n}^{\prime}$. If $\lambda_{n}=1$, choose $i, j \in \mathbb{N}$ such that $\varphi\left(i, j, s_{n}\right)=0$, and set $x_{n}=q_{i}, x_{n}^{\prime}=q_{j}$; then

$$
\rho\left(q_{i}, q_{j}\right)<\frac{1}{s_{n}}<\frac{2}{\varepsilon n},
$$

$x_{n} \succ a_{1}$, and $b_{1} \succ x_{n}^{\prime}$. Since, clearly, $\rho\left(x_{n}, x_{n}^{\prime}\right) \rightarrow 0$ as $n \rightarrow \infty$, it follows from the uniform sequential continuity of $\succ$ that there exists $N$ such that for each $n \geqslant N$, either $a_{1} \succ x_{n}$ or $x_{n}^{\prime} \succ b_{1}$. If $n \geqslant N$ and $\lambda_{n}=1$, then $x_{n} \succ a_{1}$ and $b_{1} \succ x_{n}^{\prime}$, a contradiction. Hence $\lambda_{n}=0$, and therefore $n^{-1} s_{n}<\varepsilon$, for all $n \geqslant N$. Since the sequence $\left(s_{n}\right)_{n \geqslant 1}$ and $\varepsilon>0$ are arbitrary, we conclude that $S$ is indeed pseudobounded.

Now applying BD- $\mathbb{N}$, we obtain a positive integer $K$ such that $k<K$ for each $k \in S$. Given $x, x^{\prime} \in X$ with $\rho\left(x, x^{\prime}\right)<1 /(K+1)$, suppose that $x>a_{0}$ and $b_{0} \succ x^{\prime}$. Then, by the density of the sequence $\left(q_{n}\right)_{n \geqslant 1}$, there exist $i, j$ such that $q_{i} \succ a_{0} \succ b_{0} \succ q_{j}$ and $\rho\left(q_{i}, q_{j}\right)<1 /(K+1)$. Hence $\varphi(i, j, K) \neq 1$, so $\varphi(i, j, K)=0$ and therefore $K \in S$, which is absurd. We conclude that

$$
\neg\left(x \succ a_{0} \wedge b_{0} \succ x^{\prime}\right),
$$

and hence that either $a \succ x$ or $x^{\prime} \succ b$. This completes the proof of Theorem 3.1.

Our last result is a converse to Theorem 3.1.

Theorem 3.2 Suppose that every uniformly sequentially continuous, order-dense preference relation represented by a utility function on a complete, separable metric space is uniformly continuous. Then BD- $\mathbb{N}$ holds.

Proof Let $S$ be an inhabited, countable, pseudobounded subset of $\mathbb{N}$. We may assume that $0 \in S$. Let

$$
Z=\{0\} \cup\left\{2^{-m}: m \in S\right\} .
$$

Following Ishihara ([16], Proposition 1), we can construct a mapping $f: Z \rightarrow[0,1]$ such that

(a) if $x \in Z$ and $f(x)=0$, then $x=0$, and

(b) if $x \in Z$ and $f(x)=1$, then $x=2^{-m}$ for some $m \in S$.

Let $X$ be the closure of $Z$ in $\mathbb{R}$; then $X$ is both separable and complete. In view of [13], Proposition 9, and [16], Proposition 1, we can extend $f$ to a uniformly sequentially continuous mapping $u$ of $X$ into $\{0,1\}$. Also defining

$$
u(x)=x-2 \quad(2 \leqslant x \leqslant 3),
$$

we obtain a uniformly sequentially continuous mapping $u$ from the complete space $X \cup[2,3]$ into $[0,1]$. Let $\succ$ be the corresponding preference relation, defined at (1). Then $\succ$ is uniformly sequentially continuous. Moreover, it is order dense: for if $x, y \in X$ and $u(x)>u(y)$, then, since $f(2)=0$ and $f(3)=1$, the intermediate-value theorem tells us that there exists $z \in[2,3]$ such that $u(x)>u(z)>u(y)$ and therefore $x \succ z \succ y$. Suppose that $\succ$ is uniformly continuous. Since $u(1)=1>0=u(0)$, there exists a positive integer $N$ such that if $x, x^{\prime} \in X \cap[0,1]$ and $\left|x-x^{\prime}\right|<2^{-N}$, then either $u(x)>0$ or $u\left(x^{\prime}\right)<1$, and therefore either $u(x)=1$ or $u\left(x^{\prime}\right)=0$. If $n>N$ and $n \in S$, then $2^{-n} \in X$ and $u\left(2^{-n}\right)=1$; but since $u(0)=0$ and $\left|2^{-n}-0\right|<2^{-N}$, we have either $u\left(2^{-n}\right)=0$ or $u(0)=1$. This is absurd, so $n \leqslant N$ for all $n \in S$; whence $S$ is bounded. 
We see from Theorems 3.1 and 3.2 that BD- $\mathbb{N}$ is equivalent to the passage from uniform sequential continuity to uniform continuity for order-dense preference relations on separable metric spaces.

Our main theorems have analogues for various types of (equi)continuity for sequences of functions; so it is reasonable to ask how, if at all, the use of a utility function would assist in their proofs.

Using the appropriate continuity notions, in [9] we proved inter alia the equivalence of the following:

(a) Every pointwise equicontinuous sequence of functions from $[0,1]$ into a metric space is uniformly sequentially continuous.

(b) Every pointwise continuous mapping of $[0,1]$ into $\mathbb{R}$ is uniformly sequentially continuous.

(c) $[0,1]$ has the anti-Specker property.

Suppose that, in Theorems 2.1 and 2.2, the preference relation acts on $[0,1]$ and is represented by a pointwise continuous utility function (so it is order-dense). Then the implication from (c) to (b) suffices to establish the conclusion of Theorem 2.1, and that from (b) to (c) the conclusion of Theorem 2.2.

On the other hand, putting together results from [9] and [13], we obtain the equivalence of these five statements:

(d) Every uniformly sequentially equicontinuous sequence of mappings of a separable metric space into a metric space is uniformly equicontinuous.

(e) Every uniformly sequentially continuous mapping of a complete separable metric space into a metric space is uniformly continuous.

(f) Every uniformly sequentially continuous mapping of a separable metric space into a metric space is uniformly continuous.

(g) Every uniformly sequentially continuous mapping of a separable metric space into a metric space is pointwise continuous.

(h) BD-N.

The implication from (h) to (f) gives us Theorem 3.1 under the additional hypothesis that the preference relation is represented by a uniformly sequentially continuous utility function. (It appears, at first sight, that we have dispensed with the order-density hypothesis here; however, that hypothesis actually holds in view of the equivalence of (h) and (g)). On the other hand, the implication from (e) to (h) gives us a weak form of Theorem 3.2: one with the words order-dense removed from the hypothesis.

Thus, in the presence of a utility function with an appropriate continuity condition, when the preference relation acts on $[0,1]$, Theorems 2.1-3.1, and a weaker form of Theorem 3.2, follow from work in [9] and [13]. However, our present theorems have the advantage of not needing utility functions of any type, let alone continuous ones.

\section{References}

[1] Aczel, P., and M. Rathjen, Notes on Constructive Set Theory, Report No. 40, Institut Mittag-Leffler, Royal Swedish Academy of Sciences, Stockholm, 2001. 97

[2] Baroni, M. A., and D. S. Bridges, "Continuity properties of preference relations," forthcoming in Electronic Notes in Theoretical Computer Science, 2007. 97, 98, 102 
[3] Berger, J., and D. S. Bridges, "A fan-theoretic equivalent of the antithesis of Specker's theorem," Koninklijke Nederlandse Akademie van Wetenschappen. Indagationes Mathematicae. New Series, vol. 18 (2007), pp. 195-202. 100

[4] Bishop, E. A., Foundations of Constructive Analysis, McGraw-Hill Book Co., New York, 1967. Zbl 0183.01503. MR 0221878. 97

[5] Bishop, E. A., and D. S. Bridges, Constructive Analysis, vol. 279 of Grundlehren der Mathematischen Wissenschaften, Springer-Verlag, Berlin, 1985. Zbl 0656.03042. MR 804042. 97

[6] Bridges, D. S., "The constructive theory of preference relations on a locally compact space," Koninklijke Nederlandse Akademie van Wetenschappen. Indagationes Mathematicae, Series A, vol. 92 (1989), pp. 141-65. Zbl 0679.03027. MR 1005047. 97, 100

[7] Bridges, D. S., "The constructive theory of preference relations on a locally compact space. II," Mathematical Social Sciences, vol. 27 (1994), pp. 1-9. Zbl 0879.90008. MR 1267685. 97

[8] Bridges, D. S., "Constructive methods in mathematical economics," pp. 1-21 in Mathematical Utility Theory (Essen, 1997), edited by G. Herden, Springer, Vienna, 1999. Zeitschrift für Nationalökonomie, Supplement 8, pp. 1-21 (1999). Zbl 0946.91005. MR 1716351. 97, 100

[9] Bridges, D. S., "Constructive notions of equicontinuity," University of Canterbury, preprint, 2007. 100, 101, 104

[10] Bridges, D. S., and F. Richman, Varieties of Constructive Mathematics, vol. 97 of London Mathematical Society Lecture Note Series, Cambridge University Press, Cambridge, 1987. Zbl 0618.03032. MR 890955. 99

[11] Bridges, D. S., and F. Richman, "A recursive counterexample to Debreu's theorem on the existence of a utility function," Mathematical Social Sciences, vol. 21 (1991), pp. 179-82. Zbl 0734.90011. MR 1113825. 100

[12] Bridges, D. S., and L. S. Vîţă, Techniques of Constructive Analysis, Universitext. Springer, New York, 2006. Zbl 1107.03065. MR 2253074. 97

[13] Bridges, D. S., H. Ishihara, P. M. Schuster, and L. S. Vîţă, "Strong continuity implies uniform sequential continuity," Archive for Mathematical Logic, vol. 44 (2005), pp. 88795. Zbl 1088.03048. MR 2192160. 98, 99, 103, 104

[14] Debreu, G., "Representation of a preference ordering by a numerical function," pp. 15965 in Decision Processes, edited by R. M. Thrall, C. H. Coombs, and R. L. Davies, John Wiley \& Sons Inc., New York, 1954. Zbl 0058.13803. MR 0066616. 100

[15] Friedman, H. M., "Set theoretic foundations for constructive analysis," Annals of Mathematics. Second Series, vol. 105 (1977), pp. 1-28. Zbl 0353.02014. MR 0434784. 97

[16] Ishihara, H., "Continuity properties in constructive mathematics," The Journal of Symbolic Logic, vol. 57 (1992), pp. 557-65. Zbl 0771.03018. MR 1169194. 99, 102, 103

[17] Ishihara, H., "Sequentially continuity in constructive mathematics," pp. 5-12 in Combinatorics, Computability and Logic (Constanţa, 2001), edited by C. S. Calude, M. J. Dinneen, and S. Sburlan, Springer Series in Discrete Mathematics and Theoretical Computer Science, Springer, London, 2001. Zbl 0987.03053. MR 1934817. 102 
[18] Ishihara, H., and S. Yoshida, "A constructive look at the completeness of the space $D(\mathbb{R})$," The Journal of Symbolic Logic, vol. 67 (2002), pp. 1511-19. Zbl 1053.03036. MR 1955249. 102

[19] Lietz, P., From Constructive Mathematics to Computable Analysis via the Realizability Interpretation, Ph.D. thesis, Technische Universität Darmstadt, Darmstadt, 2004. 102

[20] Myhill, J., “Constructive set theory," The Journal of Symbolic Logic, vol. 40 (1975), pp. 347-82. Zbl 0314.02045. MR 0381941. 97

[21] Specker, E., "Nicht konstruktiv beweisbare Sätze der Analysis," The Journal of Symbolic Logic, vol. 14 (1949), pp. 145-58. Zbl 0033.34102. MR 0031447. 100

[22] Troelstra, A. S., and D. van Dalen, Constructivism in Mathematics. An Introduction, Vol. I and II, vols. 121 and 123 of Studies in Logic and the Foundations of Mathematics, North-Holland Publishing Co., Amsterdam, 1988. Zbl 0661.03047. MR 966421. 97

\section{Acknowledgments}

The author thanks the referee for several suggestions, the implementation of which made substantial improvements to the paper.

Department of Mathematics \& Statistics

University of Canterbury

Private Bag 4800

Christchurch

NEW ZEALAND

d.bridges@math.canterbury.ac.nz 